\title{
A convenient method for simultaneous quantification of multiple phytohormones and metabolites: application in study of rice-bacterium interaction
}

\author{
Hongbo Liu, Xianghua Li, Jinghua Xiao and Shiping Wang
}

\begin{abstract}
Background: Simultaneous analysis of multiple functional-related phytohormones and their metabolites will improve our understanding of interactions among different hormones in the same biologic process.

Results: A method was developed for simultaneous quantification of multiple phytohormones, abscisic acid, indole-3-acetic acid (IAA), jasmonic acid (JA), and salicylic acid, hormone conjugates, IAA-aspartic acid, JA-isoleucine, and methyl JA, and phytoalexins, momilactone A, naringenin, and sakuranetin. This method combines a convenient procedure for preparing filtrated crude extracted samples and a sensitive quantification assay using ultra fast liquid chromatography-electrospray ionization tandem mass spectrometry (UFLC-ESI-MS). With this method, we determined the dynamic profiles of defense-related phytohormones, hormone metabolites, and phytoalexins in the interaction of rice with Xanthomonas oryzae pv. oryzae (Xoo), which causes bacterial blight, one of the most devastating diseases of rice worldwide.

Conclusion: This UFLC-ESI-MS method is convenient, sensitive, reliable, and inexpensive for quantification of multiple phytohormones and metabolites compared to current methods. The results obtained by application of this method in studying rice-bacterial interaction provide a basis for understanding the molecular mechanisms of rice defense responses.
\end{abstract}

Keywords: Abscisic acid, defense, LC-ESI-MS, indole-3-acetic acid, jasmonic acid, phytoalexin, salicylic acid

\section{Background}

Phytohormones are essential for the regulation of diverse physiologic processes of plants, including development, growth, reproduction, and responses to biotic and abiotic stresses. Plant-produced hormones include nonpeptide hormones, abscisic acid (ABA), auxin or indole-3-acetic acid (IAA, the major form of auxin in most plants), brassinosteroids, cytokinins, ethylene, gibberellins, jasmonic acid (JA), nitric oxide, salicylic acid (SA), and strigolactones, and peptide hormones [1,2]. The nonpeptide phytohormones are structurally unrelated small molecules. Phytohormones act as signal

\footnotetext{
* Correspondence: swang@mail.hzau.edu.cn
National Key Laboratory of Crop Genetic Improvement, National Center of

* Correspondence: swang@mail.hzau.edu.cn Plant Gene Research (Wuhan), Huazhong Agricultural University, Wuhan 430070, China
}

(c) 2012 Liu et al; licensee BioMed Central Ltd. This is an Open Access article distributed under the terms of the Creative Commons Attribution License (http://creativecommons.org/licenses/by/2.0), which permits unrestricted use, distribution, and reproduction in any medium, provided the original work is properly cited. low concentration. The homeostasis of these hormones is tightly controlled between the biosynthetic and metabolic pathways. The metabolism of nonpeptide phytohormones is generally categorized into three types of reactions: hydroxylation, oxidation, and conjugation $[3,4]$. For example, hydroxylation of JA results in partial biologically active 12-OH-JA and hydroxylation of ABA generates biologically active 7'-OH ABA, 8'-OH ABA, and 9'-OH ABA [3,5]. Cytokinin can be inactivated by oxidation [6]. The formation of hormone conjugates may generate different forms of active hormones, inactive storage hormones, or intermediates for catabolism, such as the active JA-isoleucine (Ile) and methyl JA (MeJA), the inactive storage IAA-alanine, and the intermediate IAA-aspartic acid (Asp) [7-9]. A tiny or small 
amount of variation in the concentration of a phytohormone may change physiologic activity, although the roles of these hormones in different biologic processes still remain to be elucidated [10]. Thus, quantification of the concentrations of hormones and hormone metabolites is frequently applied in the study of the molecular regulations of different biologic processes.

Accumulating evidence suggests that multiple phytohormones often mediate the same biologic process by additive, synergistic, or antagonistic actions, whereas each type of hormone has a characteristic biologic effect $[2,11,12]$. For example, plant-pathogen interactions result in changes in the level of various phytohormones $[10,13]$. SA, JA, and ethylene are well-known signal molecules in plant immunity. Although auxin has a pivotal function in plant development and growth, this hormone also has a position in plant-pathogen interactions [14]. Auxin makes plants susceptible to some biotrophic and hemibiotrophic pathogens [15-17] but resistant to necrotrophic pathogens $[18,19]$. The auxindependent pathway antagonistically interacts with the SA-dependent pathway in the Arabidopsis-pathogen interaction [20] but shares many commonalities with the JA-dependent pathway $[2,14]$. ABA signaling in abiotic stress responses has been intensively studied [21]. In addition, this hormone is also a player in hostpathogen interactions [22]. ABA can promote disease in some cases and promote defense response in other cases by antagonistic interaction with SA and JA/ethylene or synergistic interaction with JA [22,23].

Because of the complex crosstalk among different hormone signaling pathways and the multifaceted roles of these signaling molecules in a biologic process, simultaneous quantification of multiple hormones and their metabolites in the same sample will facilitate the understanding of the interactions of different hormones. The interactions of different phytohormones frequently occur in a localized tissue in certain biologic processes $[2,11,12]$, which may limit the quantity of tissue samples. Thus, a highly sensitive analytical method is essential for determining the quantitative variation of hormones in the samples that have low concentrations of hormones. Several reports have been published outlining the simultaneous quantification of multiple hormones. Müller et al. [24] reported a multiplex gas chromatography (GC)tandem mass spectrometry (MS/MS) approach for simultaneous quantification of acidic phytohormones and related compounds, ABA, IAA, JA, SA, and 12-oxophytodienoic acid. However, GC-MS/MS analysis requires a complicated sample preparation procedure including separation, purification, and derivatization. Two groups used high-performance liquid chromatography (HPLC)-electrospray ionization (ESI)-MS/MS system to simultaneously quantify acidic hormones ABA,
IAA, JA, and SA $[25,26]$. Further study reported that using HPLC-ESI-MS/MS allows simultaneous quantification of both acidic and basic hormones, IAA, JA, SA, and zeatin, and related metabolites [27]. Kojima et al. [28] reported simultaneous quantification of different molecular species of ABA, cytokinins, gibberellins, and IAA using an ultra-performance liquid chromatographyESI-MS/MS technique after purifying the samples by solid-phase extraction and chemical derivatization. Although more small molecules were quantified in this LC-MS method [28], the sample preparation procedure is complicated compared to the one used by Durgbanshi et al. [25], Forcat et al. [26], and Pan et al. [27].

Plants also produce secondary metabolites, known as phytoalexins that serve as antibiotics in response to biotic and abiotic stresses. Phytoalexins contribute to plant basal immunity and accumulate around the infection sites soon after the infection of pathogens [29]. However, the relationship between the pathogen infectionresponsive crosstalk of different hormone signaling pathways and the accumulation of phytoalexins is poorly understood.

In this study, we established an ultra fast liquid chromatography (UFLC)-ESI-MS/MS-based technique for quantitative analysis of small molecules including phytohormones, hormone metabolites, and phytoalexins. This technique incorporating a convenient sample preparation procedure is sensitive and reliable for simultaneous quantification of multiple analytes in a small amount of tissue. Using this technique, we analyzed the putative interactions of several phytohormones, hormone metabolites, and phytoalexins in rice-bacterium interactions.

\section{Results and Discussion}

\section{Optimizing quantification conditions}

The mass spectrometer was operated in a Q1 scan mode to obtain the mass spectra (MS1) and in a product ion scan mode to obtain the product ion spectra (MS2) of the $[\mathrm{M}-\mathrm{H}]$ or $[\mathrm{M}+\mathrm{H}]$ ions. The most abundant precursor and product ion fragmentation for each analyte were selected to carry out the multiple reaction monitoring (MRM) scans. To produce maximal signal for each precursor-product ion transition, different parameters of collision energy, declustering potential, entrance potential, collision cell exit potential, and nitrogen flow rate were tested. Table 1 summarizes the optimized analyzing conditions for the precursor and characteristic product ion of each analyte.

\section{Quantification using UFLC-ESI-MS/MS}

Elutes were monitored by a series of MRM scans with scan mode change $700 \mathrm{~ms}$ as settle time and $50 \mathrm{~ms}$ as dwell time for each precursor-to-product transition. The analyte and its internal standard co-elute in the same or 
Table 1 Optimized MS/MS conditions for quantifying phytohormones and metabolites

\begin{tabular}{|c|c|c|c|c|c|c|c|}
\hline Analyte $^{a}$ & $\begin{array}{c}\text { Scan } \\
\text { mode }^{b}\end{array}$ & $\begin{array}{l}\text { Precursor ion } \\
(\mathrm{m} / \mathrm{z}) \text { Q1 }\end{array}$ & $\begin{array}{l}\text { Product ion } \\
(\mathrm{m} / \mathrm{z}) \mathrm{Q} 3\end{array}$ & $\begin{array}{l}\text { Declustering } \\
\text { potential }(\mathrm{V})^{\mathrm{c}}\end{array}$ & $\begin{array}{c}\text { Entrance } \\
\text { potential }(\mathrm{V})^{\mathrm{c}}\end{array}$ & $\begin{array}{c}\text { Collision } \\
\text { energy }(\mathrm{V})^{\mathrm{c}}\end{array}$ & $\begin{array}{l}\text { Collision cell exit } \\
\text { potential (V) }\end{array}$ \\
\hline SA & - & 136.9 & 92.9 & -47 & -6 & -22 & -3 \\
\hline$N A A^{a}$ & - & 185 & 141.0 & -25 & -3 & -12 & -7 \\
\hline $\mathrm{ABA}$ & - & 263 & 153.0 & -60 & -5 & -15 & -8 \\
\hline${ }^{2} \mathrm{H}_{6} \mathrm{ABA} \mathrm{A}^{\mathrm{a}}$ & - & 269 & 159.1 & -57 & -5 & -16 & -8 \\
\hline$J A$ & - & 209 & 59.0 & -60 & -6 & -24 & -8.6 \\
\hline $\mathrm{DHJA} \mathrm{A}^{\mathrm{a}}$ & - & 211 & 59.0 & -61 & -9 & -25 & -8 \\
\hline JA-lle & - & 322.2 & 130.0 & -67 & -7.5 & -32 & -7 \\
\hline MeJA & + & 225.2 & 151.2 & 52 & 4 & 16 & 8 \\
\hline IAA & - & 173.9 & 129.9 & -53 & -4 & -13 & -15 \\
\hline IAA-Asp & - & 289 & 131.9 & -66 & -4 & -25 & -6 \\
\hline$D_{2}-I A A^{a}$ & - & 176 & 131.9 & -48 & -4 & -14 & -6 \\
\hline Naringenin & - & 271.2 & 151.0 & -76 & -10 & -25 & -8 \\
\hline Sakuranetin & - & 285.1 & 119.0 & -85 & -10 & -44 & -5 \\
\hline $\begin{array}{c}\text { Momilactone } \\
\text { A }\end{array}$ & + & 315.2 & 271.3 & 82 & 4 & 18 & 6 \\
\hline
\end{tabular}

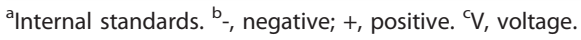

similar time. Because of their different precursor-to-product ion transitions, we specifically detect analyte and its internal standard by extracted ion current from total ion current that combines positive or negative scans. To ensure that the elute peak was the analyte or internal standard, we quantified the elute peak by MRM-triggered information-dependent acquisition-enhanced product ion analysis. The representative LC chromatograms and MS fragmentation patterns of analytes are presented in Additional file 1, Figures S1 and S2. The retention times of analytes were in a range of 8.28 to $19.40 \mathrm{~min}$ (Additonal file, Table S1).

To verify the reproducibility of analytes under optimum UFLC-ESI-MS/MS condition, the retention time and peak areas was investigated by repeated injection ( $\mathrm{n}$ $=5$ ) of a mixture of the standards at a concentration of $10 \mathrm{ng} / \mathrm{ml}$ for each standard. The relative standard deviations (RSDs) of the retention times for all the analytes were in a range of $0.0-0.29 \%$ (Table 2). The RSDs of peak areas obtained for all the analytes were in a range of $0.73-4.06 \%$ (Table 2).

We used the detection limit (signal/noise) $>10$ as a threshold for quantification of each analyte. The limit of quantification was 0.01 to $0.1 \mathrm{ng} / \mathrm{ml}$ for different analyte (Table 2). A calibration curve generated by a series of fixed amounts of standard was used to determine the amount of each analyte in the sample. The concentration ranges of standards used for generating the calibration curves were 1-100 $\mathrm{ng} / \mathrm{ml}$ (Table 2). The quantities of the analytes in the samples ranged from $1 \mathrm{ng} / \mathrm{g}$ to $2000 \mathrm{ng} / \mathrm{g}$ fresh weight tissue. The calibration curves were linear in the concentration ranges of the analytes.

\section{Comparison of different sample preparation procedures}

To determine the efficiency of analyte extraction from tissues, we quantified each analyte by UFLC-ESI-MS/MS

Table 2 Calibration curve and other related data for quantifying phytohormones and metabolites

\begin{tabular}{|c|c|c|c|c|c|}
\hline \multirow[t]{2}{*}{ Analyte } & \multicolumn{2}{|l|}{ Calibration curve } & \multirow{2}{*}{$\begin{array}{c}\text { limit of quantification } \\
(\mathrm{ng} / \mathrm{ml})\end{array}$} & \multicolumn{2}{|c|}{ Reproducibility (RSD) } \\
\hline & Standard concentration range $(\mathrm{ng} / \mathrm{ml})$ & Correlation coefficient & & Retention time (\%) & Peak area (\%) \\
\hline IAA & $1-40$ & 0.9998 & 0.05 & 0 & 0.73 \\
\hline$J A$ & $1-100$ & 0.9992 & 0.01 & 0 & 2.42 \\
\hline$J A-\| l e$ & $1-100$ & 0.9997 & 0.01 & 0.26 & 2.75 \\
\hline$A B A$ & $1-100$ & 0.9983 & 0.01 & 0 & 2.29 \\
\hline SA & $1-50$ & 0.9995 & 0.10 & 0 & 3.11 \\
\hline IAA-Asp & $1-50$ & 0.9902 & 0.10 & 0.29 & 1.77 \\
\hline MeJA & $1-50$ & 0.9932 & 0.10 & 0.25 & 2.56 \\
\hline Momilactone A & $5-100$ & 0.9987 & 0.10 & 0 & 3.00 \\
\hline Sakuranetin & $5-100$ & 0.9993 & 0.10 & 0 & 2.51 \\
\hline Naringenin & $5-100$ & 0.9917 & 0.10 & 0 & 4.06 \\
\hline
\end{tabular}


after each round of analyte extraction in preliminary experiments. After two rounds of analyte extraction, no target analyte could be detected in the purified samples. This result suggests that two rounds of analyte extraction are enough for extracting all the target analytes from tissues. Thus samples were prepared by two rounds of analyte extraction in the following experiments.

Filtration of crude sample extracts before quantification will prolong the lifetime of column in the LC-SEIMS/MS system, although phytohormones in crude plant extracts can be quantified [26,27]. To ascertain whether different types of filters used for filtration of crude extracted samples would influence analyte recovery, the sample extraction buffer containing standard phytohormones and metabolites was filtrated with either nylon filter or cellulose filter before quantification. The recovery rates of standard phytohormones and metabolites from nylon filter-filtrated samples were ranged from $90 \%$ to $95 \%$, and the recovery rates of these standards from cellulose filter-filtrated samples were ranged from $86 \%$ to $94 \%$ (Additional file 1, Table S1). Although the two types of filters had no significantly different influence $(P>0.05)$ on analyte recovery, the cellulose filter broke frequently during filtration. Thus nylon filter is more suitable for sample preparation in the present experimental condition and was used in the following sample preparation. Durgbanshi et al. [25] reported simultaneous quantification of ABA, IAA, and JA from filtrated plant samples. However, our sample preparation method allows simultaneous quantification multiple phytohormones (ABA, IAA, JA, and SA), hormone metabolites (IAA-Asp, JA-Ile, and MeJA), and phytoalexins (momilactone $\mathrm{A}$, naringenin, and sakuranetin).

The recovery rates of analytes in rice samples were determined by calculating the recovery of internal standards. The recovery of each internal standard was calculated based on the ratio of peak area that was obtained from the extraction of internal standard added to plant samples and the peak area of the same amounts of internal standards measured directly. The recovery rates of internal standards for ABA, IAA, IAA-Asp, JA, JA-Ile, MeJA, and SA after two rounds of extraction ranged from $90 \%$ to $95 \%$ in rice samples (Additional file 1, Table S1). Because no commercial internal standards are available for phytoalexins, we can not calculate the recovery rates of phytoalexins in rice samples. However, the recovery rates of phytohormones and their metabolites in rice samples showed no significant difference $(P$ $>0.05)$ from the recovery rates of corresponding standards (Additional file 1, Table S1), we argue that the recovery rates of phytoalexins in rice sample might be approximately larger than $90 \%$. These results suggest that the procedure for sample preparation has a good sample recovery and it can be used for quantification of analytes with different polarity.

The samples for quantifying phytohormones are frequently prepared by solid-phase extraction, which purifies sample using a C-bound 18 silica column on the basis of reversed-phase interaction [16,28,30,31]. To ascertain the reliability and sensitivity of our crude extraction procedure for quantifying analytes, grinded powdery samples collected from the leaves of indica rice variety Minghui 63 (Oryza sativa ssp. indica) and japonica rice variety Zhonghua 11 (O. sativa ssp. japonica) were divided into two sets. One set of samples was extracted by crude extraction and the other set by solidphase extraction. Different phytohormones, ABA, IAA, JA, and SA, in these samples were quantified (Table 3). The quantities of the four hormones in the two sets of samples showed no significant difference from each other $(P>0.05)$. These results suggest that the filtratedcrude extract prepared by our sample preparation procedure can be used for UFLC-ESI-MS/MS analysis.

\section{Differential hormone and metabolite profiling between resistant and susceptible reactions}

Plants protect themselves from pathogen invasion via a complicated signal transduction network. Some phytohormones and metabolites function either as a positive or a

Table 3 Comparison of two sample extraction methods for phytohormone quantification

\begin{tabular}{|c|c|c|c|c|c|}
\hline \multirow[t]{2}{*}{ Analyte } & \multirow[t]{2}{*}{ Sample extraction } & \multicolumn{2}{|c|}{ Zhonghua 11} & \multicolumn{2}{|c|}{ Minghui 63} \\
\hline & & Concentration $(\mathrm{ng} / \mathrm{g})^{\mathrm{a}}$ & $P$ & Concentration $(\mathrm{ng} / \mathrm{g})^{\mathrm{a}}$ & $P$ \\
\hline \multirow[t]{2}{*}{$A B A$} & crude extraction & $38.53 \pm 2.92$ & 0.25 & $20.35 \pm 1.16$ & 0.42 \\
\hline & solid-phase extraction & $35.66 \pm 0.92$ & & $21.10 \pm 0.85$ & \\
\hline \multirow[t]{2}{*}{ IAA } & crude extraction & $3.23 \pm 0.40$ & 0.55 & $9.91 \pm 0.84$ & 0.09 \\
\hline & solid-phase extraction & $3.06 \pm 0.16$ & & $8.56 \pm 0.46$ & \\
\hline \multirow[t]{2}{*}{$J A$} & crude extraction & $40.78 \pm 1.00$ & 0.18 & $115.05 \pm 9.96$ & 0.32 \\
\hline & solid-phase extraction & $43.07 \pm 2.02$ & & $122.50 \pm 4.40$ & \\
\hline \multirow[t]{2}{*}{ SA } & crude extraction & $9221.82 \pm 732.65$ & 0.61 & $3390.37 \pm 807.91$ & 0.16 \\
\hline & solid-phase extraction & $9512.12 \pm 545.10$ & & $4317.64 \pm 291.95$ & \\
\hline
\end{tabular}

${ }^{a}$ Each data point represents mean (3 replicates) \pm standard deviation. 
negative regulator or factor in this network. ABA, IAA, JA, and SA are reportedly involved in the interactions of rice and different pathogens, although the details of their interactions remains to be elucidated [16,32-34]. The IAA-Asp, which lacks IAA bioactivity, is an intermediate in an irreversible IAA deactivation pathway [35]. Enhanced rice disease resistance to both bacterial and fungal pathogens is accompanied by increased accumulation of IAA-Asp and a reduced level of IAA $[16,17]$. JA-Ile and MeJA are bioactive jasmonates involved in biotic challenges [36-38], although their roles in rice disease resistance remain to be determined. Phytoalexins are antimicrobial secondary metabolites [29]. Rice momilactone A (a terpenoid phytoalexin) and sakuranetin (a flavonoid phytoalexin) can enhance rice resistance to fungal pathogen $[39,40]$. Naringenin is also a flavonoid phytoalexin and it can inhibit the growth of both bacterial and fungal pathogens [41]. Bacterial blight caused by Xanthomonas oryzae pv. oryzae (Xoo) is one of the most devastating diseases of rice worldwide. To study the crosstalk of different phytohormones and the relationship and interactions of phytoalexins and hormone-dependent pathways, the dynamic contents of defense-related hormones, ABA, IAA, JA, and SA, their metabolites, IAA-Asp, JA-Ile, and MeJA, and phytoalexins, momilactone A, naringenin, and sakuranetin, in resistant and susceptible reactions were simultaneously quantified and compared using the established UFLC-ESI-MS/MS technique.

Rice variety Minghui 63 carrying major disease resistance genes Xa3/Xa26 and xa25 against Xoo is moderately resistant to Xoo strain PXO61, whereas variety Zhenshan 97 is susceptible to Xoo [42-45]. The lesion area caused by PXO61 infection was more than 2-fold larger in Zhenshan 97 than in Minghui 63 (Figure 1).

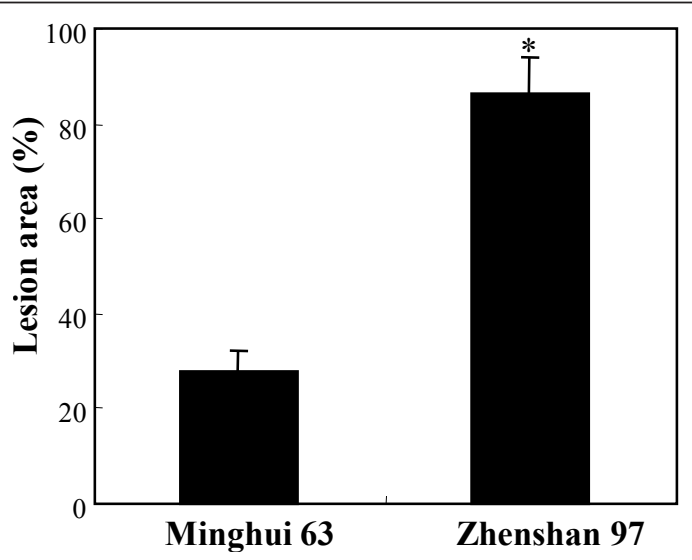

Figure 1 The response of rice varieties Minghui 63 and Zhenshan 97 to Xoo strain PXO61. Each bar represents mean (5 replicates) \pm standard deviation. The asterisk $\left(^{*}\right)$ indicates that a significant difference between Minghui 63 and Zhenshan 97 was detected at $P<0.01$.
The infected leaf tissues were collected for simultaneously quantifying target analytes by UFLC-ESI-MS/ MS. The expression patterns of genes known to be involved in hormone and phytoalexin biosynthesis were also analyzed using the same samples and compared to the analyte profiles to examine the reliability of this quantification technique.

ABA

The ABA concentration was 1.6-fold higher in resistant Minghui 63 than in susceptible Zhenshan 97 when without pathogen infection. After inoculation of Xoo strain PXO61, the ABA levels in the two rice varieties showed a similar variation pattern in that ABA was significantly increased $(P<0.05)$ at early infection, returned to basal level, and markedly increased again at $7 \mathrm{~d}$ after infection (Figure 2). However, the ABA level in Minghui 63 was 27\% lower than that in Zhenshan 97 at $7 \mathrm{~d}$ after infection. ZEP (Rice Genome Annotation Project [http://rice. plantbiology.msu.edu/] locus name: Os04g37619), encoding a zeaxanthin epoxidase, and NCED, encoding a 9-cis-epoxycarotenoid dioxygenase, are the key genes controlling ABA biosynthesis [46,47]. The three genes, ZEP, NCED1 (GenBank accession no. AY838897), and NCED3 (AY838899), were markedly induced at $7 \mathrm{~d}$ after infection with the expression level significantly higher $(P$ $<0.05$ ) in Zhenshan 97 than in Minghui 63, which was consistent with the differential accumulation of ABA at $7 \mathrm{~d}$ after infection in the two rice varieties (Figure 2). Thus, the expression patterns of these genes further confirmed the reliability of the quantification results.

$\mathrm{ABA}$ is involved in plant-pathogen interactions, but its role in these interactions is complex. In plant immunity, ABA functions as a negative signal molecule in some cases but a positive signal molecule in other cases $[22,23]$. Exogenous application of ABA compromises rice resistance to the fungal pathogen Magnaporthe oryzae $[34,48]$. However, the role of ABA in the interaction of rice and bacterial pathogen $X o o$ is not clear, although protein interaction study around key regulators of biotic response suggests that $\mathrm{ABA}$ has an important function in rice resistance to Xoo [49]. The present results show that Xoo-induced susceptible rice plants accumulated more ABA than the resistant plants. Thus, further study may target the putative negative role of $\mathrm{ABA}$ in rice-Xoo interaction.

\section{IAA}

The variation in endogenous IAA levels showed a similar pattern as ABA in the two rice varieties before and after infection with Xoo. The concentration of IAA was 2.5-fold higher in resistant Minghui 63 than in susceptible Zhenshan 97 when without pathogen infection. After Xoo infection, the IAA levels in the two rice varieties showed a similar variation pattern in that they were significantly increased $(P<0.01)$ at $12 \mathrm{~h}$ after 


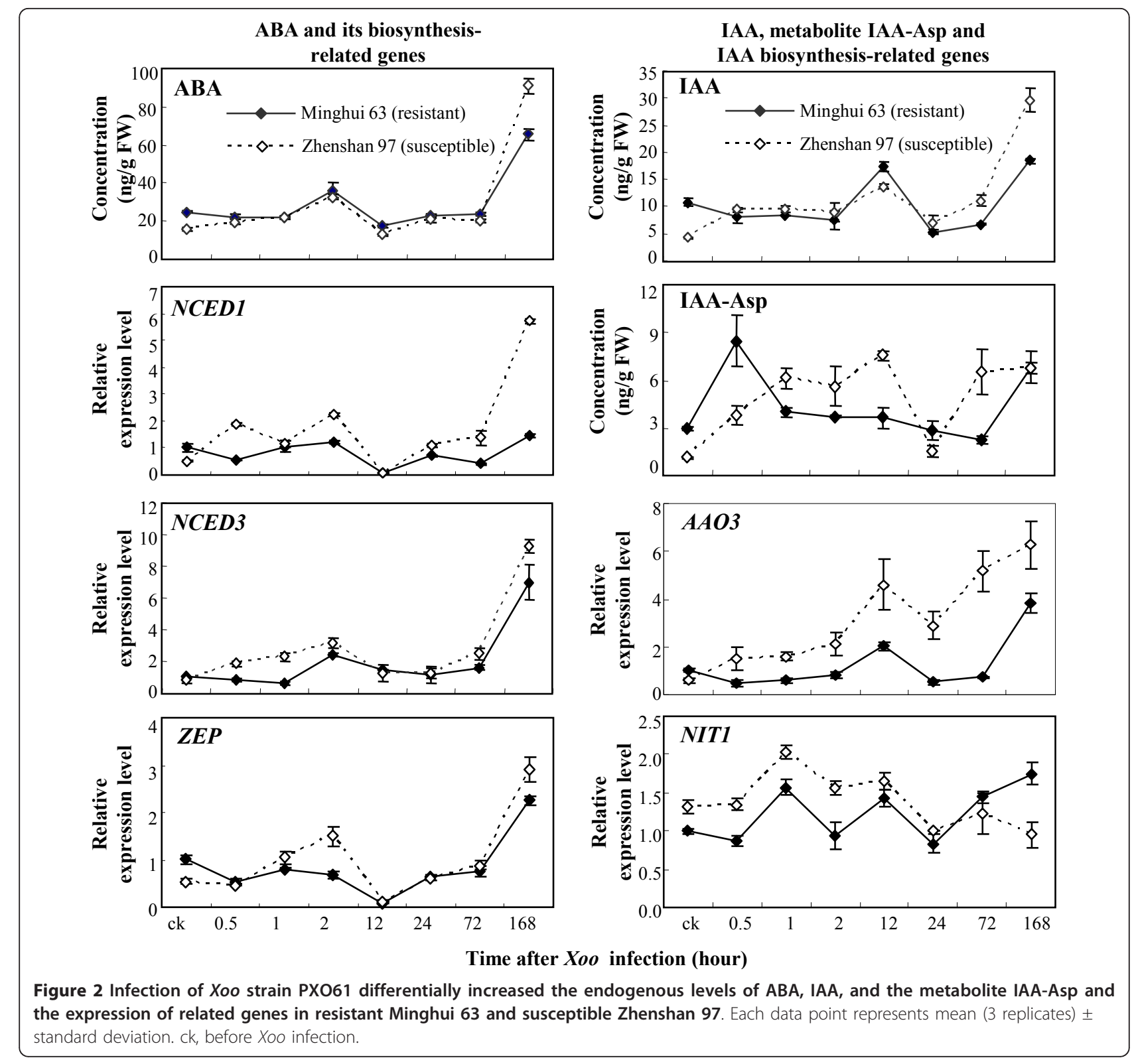

infection, returned to basal levels at 24 to $72 \mathrm{~h}$ after infection, and markedly increased again at $7 \mathrm{~d}$ after infection (Figure 2). However, the IAA level in Minghui 63 was 38\% lower than that in Zhenshan 97 at $7 \mathrm{~d}$ after infection. Consistent with the IAA level, the IAA-Asp level, the inactive form of IAA, was significantly higher $(P<0.01)$ in Minghui 63 than in Zhenshan 97 before infection and immediately (30 $\mathrm{min}$ ) after infection (Figure 2). However, the IAA-Asp level was significantly higher $(P<0.01)$ in Zhenshan 97 than in Minghui 63 at $12 \mathrm{~h}$ after infection. Indole-3-acetaldehyde oxidase (AAO) and nitrilase (NIT) are two protein families involved in the two tryptophan-dependent pathways for IAA biosynthesis in plants, respectively [7]. The increased expression of rice AAO3 (AK065990) and NIT1 (AK104033) is associated with increased accumulation of IAA in rice, suggesting that the two genes are most likely involved in IAA synthesis $[16,17]$. The expression pattern of $A A O 3$ was similar to the level of IAA in both varieties before and after Xoo infection, suggesting that AAO3 may be more closely associated with the local accumulation of IAA after infection than the NIT1 gene in the present experimental condition (Figure 2).

Exogenous treatment of rice plants with IAA promotes disease symptoms caused by both bacterial and fungal pathogens $[16,17]$. Consistent with previous results, the present results also indicate that accumulation of more 
IAA in susceptible plants is associated with disease. Interestingly, the variation of IAA level in response to Xoo infection presented a similar pattern as the variation of ABA level (Figure 2). Although many phytohormones, either as positive or negative players, are involved in plant defense signaling networks [10], no interaction between IAA signaling and ABA signaling has been reported to our knowledge. Thus, it is worth analyzing whether the two hormones function synergistically in the rice-Xoo interaction.

\section{$J A, J A$-related compound, and SA}

The JA and SA levels showed opposite patterns in the two rice varieties at some time points examined. JA concentration was 4.2-fold higher in resistant Minghui 63 than in susceptible Zhenshan 97, whereas the SA concentration was approximately 58\% lower in Minghui 63 than in Zhenshan 97 when without pathogen infection (Figure 3 ). At early infection (30 $\mathrm{min}$ to $1 \mathrm{~h}$ ), the JA levels were significantly reduced $(P<0.01)$ in Minghui 63 and increased $(P<0.01)$ in Zhenshan 97, but SA levels were significantly increased $(P<0.01)$ in Minghui 63 and reduced in Zhenshan $97(P<0.05)$. At $12 \mathrm{~h}$ after infection, the JA level was markedly increased, but the SA level returned to the basal level as compared to noninfected plants in Minghui 63, although both JA and SA levels were significantly increased $(P<0.05)$ in Zhenshan 97. At $7 \mathrm{~d}$ after infection, the JA level in Minghui 63 was significantly higher $(P<0.01)$ than that in Zhenshan 97, whereas the SA level in Minghui 63 was significantly lower $(P<0.05)$ than that in Zhenshan 97.

JA-Ile, the active form of JA, is a major regulator that controls jasmonate-responsive gene expression and production of secondary metabolites after biotic stress [37]. The variation patterns of JA-Ile levels in response to $X o o$ infection were similar to the JA patterns in the two rice varieties (Figure 3 ). The exception is that the JA-Ile levels were approximately 2 - and 16-fold higher in susceptible Zhenshan 97 than in resistant Minghui 63 at 12 and $24 \mathrm{~h}$ after infection, respectively. MeJA is the volatile methylester of JA. It activates JA-dependent defense pathway in plant response to necrotrophic pathogens and insects [36,38]. Minghui 63 had a significantly higher $(P<0.01)$ level of MeJA than Zhenshan 97 at 30 min after infection, but Zhenshan 97 had a significantly higher $(P<0.05)$ level of MeJA than Minghui 63 at 2 to $24 \mathrm{~h}$ after infection (Figure 3 ).

Lipoxygenase ( $L O X ; \mathrm{D} 14000)$ and allene oxide synthase 2 (AOS2; AY062258) regulate JA biosynthesis. Phenylalanine ammonia-lyase 1 (PAL1; X16099) is involved in SA biosynthesis by the phenylpropanoid pathway. Isochorismate synthase 1 (ICS1; AK120689) is putatively involved in SA biosynthesis in rice by the isochorismate pathway [32]. The expression of $L O X$ and AOS 2 was influenced after infection, which showed similar variation patterns as the variation of JA levels in both Minghui 63 and Zhenshan 97 (Figure 3). PAL1 and ICS1 also showed expression patterns similar to the variation of SA levels in the two rice varieties (Figure 3). The gene expression results further confirmed the reliability of the quantification data.

Previous reports suggest that multiple JA- and SAassociated pathways may be involved in rice resistance against Xoo. For example, overexpressing MPK6 or suppressing WRKY45-1 or EDR1 enhances rice resistance accompanied by activation of both JA and SA signaling $[33,50,51]$. However, overexpressing WRKY13 or suppressing OsDR10 or MPK6 enhances rice resistance that is accompanied by activation of SA signaling and suppression of JA signaling $[32,50,52,53]$. In contrast, overexpressing WRKY45-2 enhances resistance that is associated with activation of JA signaling but not SA signaling [33]. The opposite JA and SA levels at some time points of the resistance reaction as compared to their levels in susceptible reaction suggest that the JAand SA-dependent pathways may function antagonistically in rice resistance to Xoo. According to this inference and previous reports [32,33,50-53], we argue that the JA-and SA-dependent pathways may have crisscross roles in rice-Xoo interactions.

The IAA-dependent pathway may act antagonistically to the SA-dependent defense pathway in Arabidopsispathogen interaction; SA-mediated plant immunity accompanies the repression of the auxin signaling in Arabidopsis [20,54]. However, the dynamic profiling of IAA and SA after Xoo infection did not show obvious antagonistic interaction (Figures 2 and 3). Previous studies have also revealed that enhancing rice resistance to Xoo by suppressing IAA signaling does not require activation of SA signaling $[16,17]$. These results suggest that in rice-pathogen interaction, IAA signaling and SA signaling may interact in a different way from that in Arabidopsis.

\section{Phytoalexins}

We used an external standard method for quantification of phytoalexins as reported previously [55]. The concentration of momilactone A was approximately 69\% lower in resistant Minghui 63 than in susceptible Zhenshan 97 when without pathogen infection (Figure 4). However, $X o o$ infection more rapidly increased momilactone A accumulation in Minghui 63 than in Zhenshan 97. Consistent with the accumulation of momilactone A, Xoo infection also induced the expression of momilactone A biosynthesis-related genes KSL4 (AK119327) and CPS4 (AK100631) that encode syn-pimaradiene synthase and syn-CDP synthase, respectively [56,57]. The levels of sakuranetin and naringenin showed a similar pattern of variation in resistant Minghui 63 after infection (Figure 4), which is consistent with the fact that naringenin is 


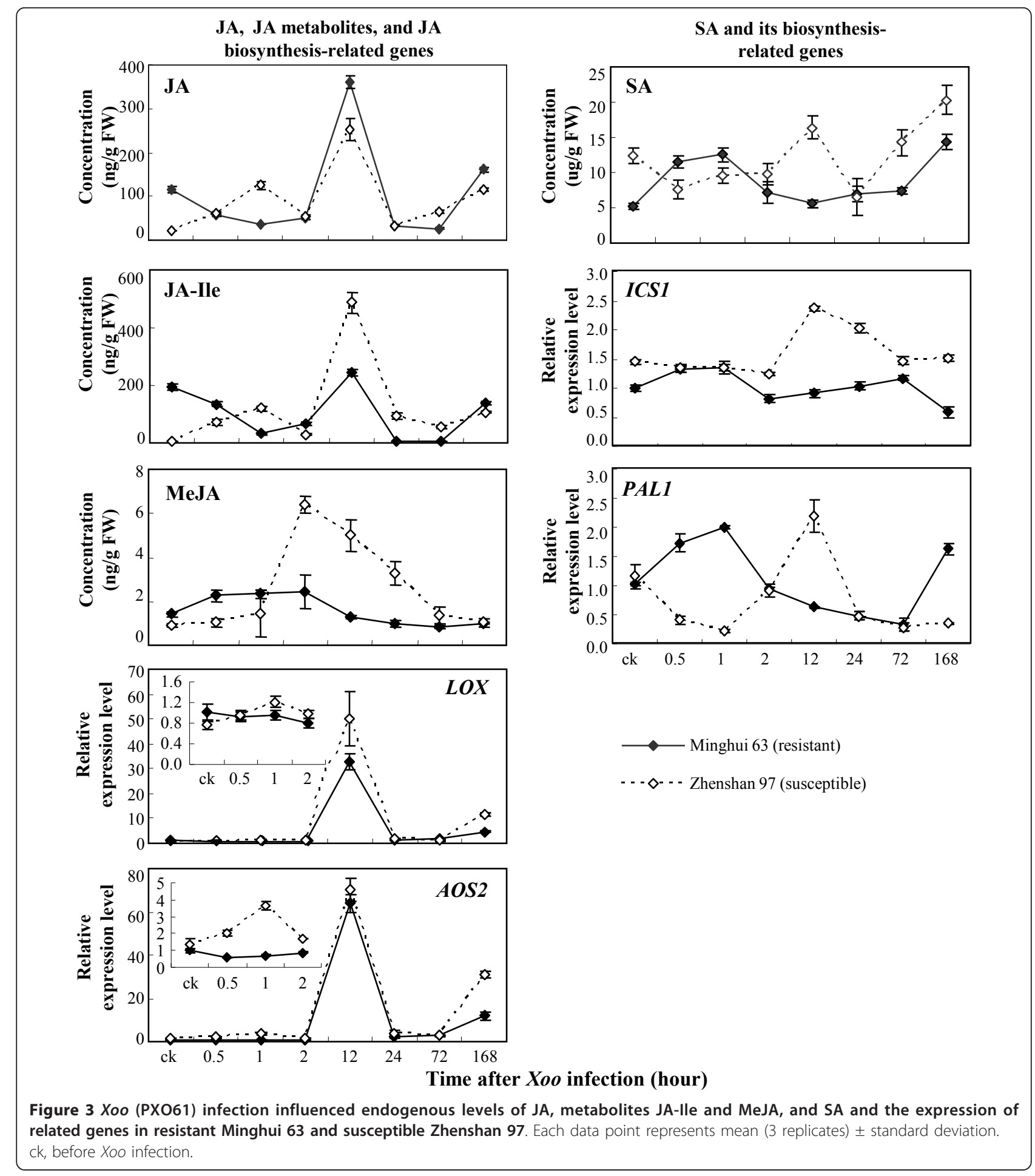

the precursor of sakuranetin [40]. After infection, the sakuranetin and naringenin levels were rapidly increased at $1 \mathrm{~h}$, return to the basal levels at $2 \mathrm{~h}$, and markedly increased again at $7 \mathrm{~d}$ in Minghui 63, but no such induction was detected in susceptible Zhenshan 97. CHS (X89859) encodes chalcone synthase that catalyzes the first step in the biosynthesis of flavonoids $[58,59]$. The expression of $\mathrm{CHS}$ showed a similar variation pattern as the patterns of sakuranetin and naringenin levels in response to Xoo infection (Figure 4).

The differentially increased accumulation of sakuranetin and naringenin in the resistance reaction but not in 


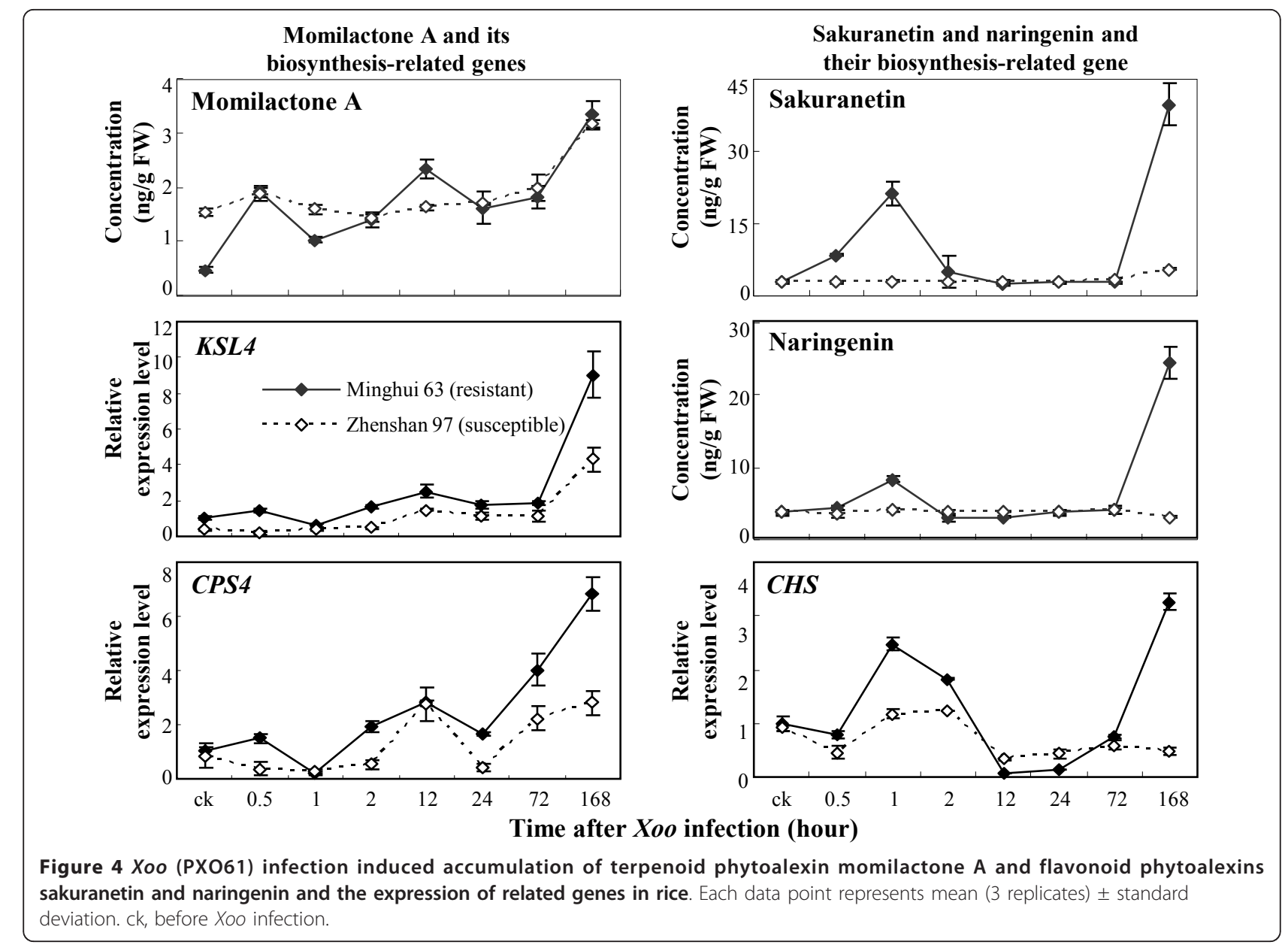

the susceptible reaction suggest that the two flavonoid phytoalexins may be involved in Xoo resistance in the present experimental conditions. This inference is also supported by the evidence that naringenin displayed growth inhibition of Xoo in culture [41]. The terpenoid phytoalexin momilactone A can inhibit the growth of rice fungal pathogen $M$. oryzae [39,40,55], but its role in rice resistance to Xoo is not clear. Although Xoo infection increased the accumulation of momilactone $\mathrm{A}$ in both resistant and susceptible rice plants, the concentrations of momilactone $\mathrm{A}$ in the two rice varieties showed no significant difference $(P>0.05)$ at $7 \mathrm{~d}$ after infection. In addition, rice WRKY13-mediated Xoo resistance does not accompany increased accumulation of momilactone A [52]. Thus, the role of momilactone A in rice-Xoo interaction remains to be elucidated.

The flavonoid phytoalexins are synthesized via a branch, which is different from the branch for SA biosynthesis, in the phenylpropanoid pathway [52]. PAL is an important enzyme upstream of the two branches in this pathway. The PAL1 showed a 4.6-fold higher expression level in resistant Minghui 63 than that in susceptible Zhenshan 97, but the SA level in the former was significantly lower $(P<0.05)$ than that in the latter at $7 \mathrm{~d}$ after infection (Figure 3). This may be explained by the fact that the phenylpropanoid pathway largely contributes to the biosynthesis of phytoalexins in the resistant reaction (Figure 4).

\section{Conclusion}

In comparison with published methods [24-28], the advantage of the present method is the convenient and inexpensive procedure for preparing filtrated crude extracted samples, which does not require a complicated procedure of sample derivatization or has less damage to the columns of the LC-ESI-MS/MS system. Furthermore, this convenient sample preparation method can be used for simultaneous quantification of multiple phytohormones and metabolites that have different polarity. By simultaneous quantification of multiple hormones and their metabolites and phytoalexins using this method and considering previous reports, we suggest that the JA- and SA-dependent pathways may function antagonistically in rice resistance to Xoo; however, the 
two pathways may have crisscross roles in the rice-Xoo interaction. In addition, at late infection, the phenylpropanoid pathway may largely contribute to the biosynthesis of flavonoid phytoalexins that can directly inhibit Xoo growth other than the biosynthesis of SA.

\section{Methods}

\section{Chemicals}

The standard IAA, IAA-Asp, JA, MeJA, and SA were purchased from Sigma-Aldrich (St. Louis, MO, USA), and ABA and JA-Ile were from OlChemIm (OlChemIm, Olomouc, Czech Republic). The internal standards were ${ }^{2} \mathrm{H}_{6}$ ABA (Olchemin) for ABA, 10-dihydro-JA (DHJA; Olchemin) for JA, JA-Ile, and MeJA, D 2 -IAA (SigmaAldrich) for IAA and IAA-Asp, and naphthalene acetic acid (NAA; internal standards) for SA. The standard momilactone A was kindly provided by Dr. Morifumi Hasegawa of College of Agriculture, Ibaraki University. The standard naringenin and sakuranetin were purchased from Extrasynthèse (Genay, France).

\section{Bacterial inoculation}

Rice varieties (Oryza sativa ssp. indica) Minghui 63 and Zhenshan 97 were inoculated with Xoo strain PXO61 by leaf-clipping method at booting (panicle development) stage [42]. Disease was scored by measuring the percentage disease area (lesion length/leaf length) at 2 weeks after inoculation. The $2-\mathrm{cm}$ leaf fragments next to bacterial infection sites were collected and stored at $-70^{\circ} \mathrm{C}$ for preparing samples for UFLC-ESI-MS/MS analysis and gene expression analyses.

\section{Sample preparation}

Samples were prepared using a modified crude extraction procedure that was originally reported by Pan et al. [27]. Three replicates of each frozen leaf sample $(\sim 100$ $\mathrm{mg}$ for each replicate) were ground to a fine power in liquid nitrogen using a mortar and pestle. Each sample was weighed into a $1.5-\mathrm{mL}$ tube, mixed with $750 \mu \mathrm{L}$ cold extraction buffer (methanol:water:acetic acid, $80: 19: 1, \mathrm{v} / \mathrm{v} / \mathrm{v})$ supplemented with internal standards, 10 ng ${ }^{2} \mathrm{H}_{6} \mathrm{ABA}, 10 \mathrm{ng} \mathrm{DHJA}, 5 \mathrm{ng} \mathrm{D}_{2}$-IAA, and $3 \mu \mathrm{g}$ NAA, vigorously shaken on a shaking bed for $16 \mathrm{~h}$ at $4^{\circ} \mathrm{C}$ in dark, and then centrifuged at 13,000 rpm for $15 \mathrm{~min}$ at $4{ }^{\circ} \mathrm{C}$. The supernatant was carefully transferred to a new $1.5-\mathrm{mL}$ tube and the pellet was remixed with $400 \mu \mathrm{L}$ extraction buffer, shaken for $4 \mathrm{~h}$ at $4^{\circ} \mathrm{C}$, and centrifuged. The two supernatants were combined and filtered using a syringe-facilitated $13-\mathrm{mm}$ diameter nylon filter with pore size $0.22 \mu \mathrm{m}$ (Nylon 66; Jinteng Experiment Equipment Co., Ltd, Tianjing, China). The filtrate was dried by evaporation under the flow of nitrogen gas for approximately $4 \mathrm{~h}$ at room temperature, and then dissolved in $200 \mu \mathrm{L}$ methanol. A aliquot of dissolved sample was further diluted 100 times using methanol for quantification of SA, because rice contains a high level of SA.

To determine whether different types of filters would influence the recovery rates of analytes, each standard (5 ng) was added into a $1.5-\mathrm{mL}$ tube, mixed with the same amount of cold extraction buffer, vigorously shaken on a shaking bed, and centrifuged as described above. The samples were then filtered using a nylon filer or a syringe-facilitated $13-\mathrm{mm}$ diameter cellulose filter with pore size $0.22 \mu \mathrm{m}$ (MCE; Navigator Lab Instrument Co., Ltd, Tianjing, China). The filtrates were dried and then dissolved in methanol as described above.

For comparison, samples were also prepared using a solid-phase extraction procedure [16]. In brief, ground sample powder was mixed with $2 \mathrm{ml}$ extraction buffer and shaken on a shaking bed for $16 \mathrm{~h}$ as for the abovedescribed preparation for crude extraction, and then centrifuged at $3500 \mathrm{~g}$ for $15 \mathrm{~min}$ at $4^{\circ} \mathrm{C}$ for collecting the supernatant. The supernatant was purified using a C18-SepPak cartridge (Waters Corporation, Milford, MA, USA) by a series of steps. The purified sample was dried by evaporation and then dissolved in $200 \mu \mathrm{L}$ of methanol as described above for preparation for the crude extraction.

\section{UFLC-ESI-MS/MS}

Liquid chromatography was carried out using a UFLC with an autosampler (Shimadzu Corporation, Kyoto, Japan). A Waters Atlantis T3 (Waters Corporation) column $(2.1 \times 150 \mathrm{~cm}, 3 \mu \mathrm{m})$ was used at ambient temperature. The injected volume of sample was $10 \mu \mathrm{L}$. The elution gradient was carried out with binary solvent system consisting of $0.02 \%$ acetic acid in $\mathrm{H}_{2} \mathrm{O}$ (solvent $\mathrm{A}$ ) and $0.02 \%$ acetic acid in $\mathrm{MeCN}$ (solvent $\mathrm{B}$ ) at a constant flow rate of $250 \mu \mathrm{L} / \mathrm{min}$. A linear gradient profile with the following proportions $(\mathrm{v} / \mathrm{v})$ of solvent B was applied: gradient profile 0 to $5 \mathrm{~min}$ and $0 \%$ of $\mathrm{B}, 5$ to $8 \mathrm{~min}$ and $0 \%$ to $16 \%$ of B, 8 to $20 \mathrm{~min}$ and $16 \%$ to $100 \%$ of B, 20 to $25 \mathrm{~min}$ and $100 \%$ of B, 25 to $28 \mathrm{~min}$ and $100 \%$ to $0 \%$ of $\mathrm{B}$, and 28 to $32 \mathrm{~min}$ with $4 \mathrm{~min}$ for re-equilibration and $0 \%$ of $\mathrm{B}$.

To diagnose the hormone precursor-to-product ion transitions, mixtures of $150 \mathrm{ng} / \mathrm{mL}$ of the standard compounds dissolved in $50 \% \mathrm{MeCN}$ were directly infused into a hybrid triple quadrupole/linear ion trap mass spectrometer (ABI 4000 Q-Trap, Applied Biosystems, Foster City, CA, USA) outfitted with an electrospray ion source. The analysis parameters were optimized for the production of characteristic precursor-to-product ion transitions in negative or positive ionization modes. ABA, IAA, IAA-Asp, JA, JA-Ile, SA, sakuranetin, naringenin, and their internal standards were scanned in the negative mode, whereas momilactone $\mathrm{A}$ and MeJA were 
analyzed in the positive mode. The mixtures of standard compounds were separated by reversed-phase UFLC and analyzed by ESI-MS/MS in the MRM mode with $50 \mathrm{~ms}$ dwell time, $5 \mathrm{~ms}$ of pause time between mass ranges, and $700 \mathrm{~ms}$ of settle time for switching polarities. The identities of phytohormones and metabolites in the crude plant extracts were confirmed by analysis of product ion fragments obtained by the hybrid triple quadrupole/linear ion trap mass spectrometer, operating in the IDA mode, with a source voltage of $4.5 \mathrm{kV}$ and source temperature of 550. In the "Enhanced Product Ion" scan mode, precursor ions were fragmented with collision energy $+25 \mathrm{kV}$ or $-25 \mathrm{kV}$ and products in the range of 50 to $500 \mathrm{~m} / \mathrm{z}$ were detected.

UFLC-ESI-MS/MS assays were repeated twice biologically, with each repetition having three replicates. Similar results were obtained in repeated experiments; only the result in one repetition was presented.

\section{Gene expression analyses}

Quantitative reverse transcription-polymerase chain reaction (qRT-PCR) was performed as described previously [32]. The gene-specific primers are listed in Additional file 1, Table S2. The expression level of actin gene was first used to standardize the RNA sample for each qRT-PCR. The expression level relative to control was then presented. For each gene, qRT-PCR assays were repeated twice biologically, with each repetition having three replicates. Similar results were obtained in repeated experiments; only the result in one repetition was presented.

\section{Statistical analysis}

The significant differences between control and treatment of the samples were analyzed by the pair-wise $t$ test installed in the Microsoft Office Excel program.

\section{Additional material}

Additional file 1: Figure S1 Representative LC chromatograms of analytes and their internal standards. Figure S2 Representative MS fragmentation patterns of analytes. Table S1 Recovery of analytes in standard-containing samples prepared using different filters and in rice samples. Table S2 Primers used for quantitative reverse-transcription-PCR analysis.

\begin{abstract}
Acknowledgements
This work was supported by grants from the National Natural Science Foundation of China (30930063) and the National Program of High Technology Development of China (2012AA100103).
\end{abstract}

\section{Authors' contributions}

HL performed LC-ESI-MS and gene expression analyses and drafted the manuscript. XL and JX provided molecular analysis support. SW contributed to data interpretation and to writing the manuscript. All authors read and approved the final manuscript.

\section{Competing interests}

The authors declare that they have no competing interests.

Received: 28 September 2011 Accepted: 15 January 2012

Published: 15 January 2012

\section{References}

1. Jun JH, Fiume E, Fletcher JC: The CLE family of plant polypeptide signaling molecules. Cell Mol Life Sci 2008, 65:743-755.

2. Santner A, Estelle M: Recent advances and emerging trends in plant hormone signalling. Nature 2009, 459:1071-1078.

3. Nambara E, Marion-Poll A: Abscisic acid biosynthesis and catabolism. Annu Rev Plant Biol 2005, 56:165-185.

4. Ljun K, Hul AK, Kowalczyk M, Marchant A, Celenza J, Cohen JD, Sandberg G: Biosynthesis, conjugation, catabolism and homeostasis of indole-3-acetic acid in Arabidopsis thaliana. Plant Mol Biol 2002, 50:309-332.

5. Miersch O, Neumerkel J, Dippe M, Stenzel I, Wasternack C: Hydroxylated jasmonates are commonly occurring metabolites of jasmonic acid and contribute to a partial switch-off in jasmonate signaling. New Phytol 2008, 177:114-127.

6. Mok DW, Mok MC: Cytokinin metabolism and action. Annu. Rev. Plant Physiol. Plant Mol Biol 2001, 52:89-118.

7. Woodward AW, Bartel B: Auxin: regulation, action, and interaction. Ann Bot (Lond) 2005, 95:707-735.

8. Gfeller A, Dubugnon L, Llechtl R, Farmer EE: Jasmonate biochemical pathway. Sci Signal 2010, 3:cm3.

9. Gfeller A, Llechtl R, Farmer EE: Arabidopsis jasmonate signaling pathway. Sci Signal 2010, 3:cm4

10. Pieterse CM, Leon-Reyes A, Van der Ent S, Van Wees SC: Networking by small-molecule hormones in plant immunity. Nat Chem Biol 2009, 5:308-316

11. Robert-Seilaniantz A, Navarro L, Bari R, Jones JD: Pathological hormone imbalances. Curr Opin Plant Biol 2007, 10:372-379.

12. Kuppusamy KT, Walcher CL, Nemhauser JL: Cross-regulatory mechanisms in hormone signaling. Plant Mol Biol 2009, 69:375-381.

13. Grant MR, Jones JDG: Hormone (dis)harmony moulds plant heath and disease. Science 2009, 324:750-752.

14. Kazan K, Manners JM: Linking development to defense: auxin in plantpathogen interactions. Trends Plant Sci 2009, 14:373-382.

15. Navarro L, Dunoyer P, Jay F, Arnold B, Dharmasiri N, Estelle M, Voinnet O, Jones JD: A plant miRNA contributes to antibacterial resistance by repressing auxin signaling. Science 2006, 312:436-439.

16. Ding $X$, Cao Y, Huang L, Zhao J, Xu C, Li X, Wang S: Activation of the indole-3-acetic acid-amido synthetase $\mathrm{GH} 3-8$ suppresses expansin expression and promotes salicylate- and jasmonate-independent basal immunity in rice. Plant Cell 2008, 20:228-240.

17. Fu J, Liu H, Li Y, Yu H, Li X, Xiao J, Wang S: Manipulating broad-spectrum disease resistance by suppressing pathogen-induced auxin accumulation in rice. Plant Physiol 2011, 155:589-602.

18. Tiryaki I, Staswick PE: An Arabidopsis mutant defective in jasmonate response is allelic to the auxin-signaling mutant axr1. Plant Physiol 2002, 130:887-894

19. Llorente F, Muskett P, Sánchez-Vallet A, López G, Ramos B, SánchezRodríguez C, Jordá L, Parker J, Molina A: Repression of the auxin response pathway increases Arabidopsis susceptibility to necrotrophic fungi. $\mathrm{Mol}$ Plant 2008, 1:496-509.

20. Wang D, Pajerowska-Mukhtar K, Culler AH, Dong X: Salicylic acid inhibits pathogen growth in plants through repression of the auxin signaling pathway. Curr Biol 2007, 17:84-1790.

21. Verslues PE, Zhu JK: Before and beyond ABA: upstream sensing and internal signals that determine $A B A$ accumulation and response under abiotic stress. Biochem Soc Trans 2005, 33:375-379.

22. Ton J, Flors $V$, Mauch-Mani B: The multifaceted role of $A B A$ in disease resistance. Trends Plant Sci 2009, 14:310-317.

23. Bari $R$, Jones JD: Role of plant hormones in plant defence responses. Plant Mol Biol 2009, 69:473-488

24. Müller A, Düchting P, Weiler EW: A multiplex GC-MS/MS technique for the sensitive and quantitative single-run analysis of acidic phytohormones and related compounds, and its application to Arabidopsis thaliana. Planta 2002, 216:44-56 
25. Durgbanshi A, Arbona V, Pozo O, Miersch O, Sancho JV, Gómez-Cadenas A: Simultaneous determination of multiple phytohormones in plant extracts by liquid chromatography-electrospray tandem mass spectrometry. J Agric Food Chem 2005, 53:8437-8442.

26. Forcat S, Bennett MH, Mansfield JW, Grant MR: A rapid and robust method for simultaneously measuring changes in the phytohormones $A B A, J A$ and SA in plants following biotic and abiotic stress. Plant Methods 2008 , 3:4-16.

27. Pan $X Q$, Welti $R$, Wang $X M$ : Simultaneous quantification of major phytohormones and related compounds in crude plant extracts by liquid chromatography-electrospray tandem mass spectrometry. Phytochemistry 2008, 69:1773-1781.

28. Kojima M, Kamada-Nobusada T, Komatsu H, Takei K, Kuroha T, Mizutani M, Ashikari M, Ueguchi-Tanaka M, Matsuoka M, Suzuki K, Sakakibara H: Highly sensitive and high-throughput analysis of plant hormones using MSprobe modification and liquid chromatography-tandem mass spectrometry: an application for hormone profiling in Oryza sativa. Plant Cell Physiol 2009, 50:1201-1214.

29. Gonzalez-Lamothe R, Mitchell G, Gattuso M, Diarra MS, Malouin F, Bouarab K: Plant antimicrobial agents and their effects on plant and human pathogens. Int J Mol Sci 2009, 10:3400-3419.

30. Edlund A, Eklof S, Sundberg B, Moritz T, Sandberg G: A microscale technique for gas chromatography-mass spectrometry measurements of picogram amounts of indole-3-acetic acid in plant tissues. Plant Physiol 1995, 108:1043-1047.

31. Kowalczyk M, Sandberg G: Quantitative analysis of indole-3-acetic acid metabolites in Arabidopsis. Plant Physiol 2001, 127:1845-1853.

32. Qiu D, Xiao J, Ding X, Xiong M, Cai M, Cao Y, Li X, Xu C, Wang S: OsWRKY13 mediates rice disease resistance by regulating defenserelated genes in salicylate- and jasmonate-dependent signaling. $\mathrm{Mol}$ Plant Microbe Interact 2007, 20:492-499.

33. Tao Z, Liu H, Qiu D, Zhou Y, Li X, Xu C, Wang S: A pair of allelic WRKY genes play opposite role in rice-bacteria interactions. Plant Physiol 2009, 151:936-948.

34. Jiang CJ, Shimono M, Sugano S, Kojima M, Yazawa K, Yoshida R, Inoue H, Hayashi N, Sakakibara H, Takatsuji H: Abscisic acid interacts antagonistically with salicylic acid signaling pathway in riceMagnaporthe grisea interaction. Mol Plant Microbe Interact 2010, 23(7):91-798.

35. Ludwig-Müller J: Auxin conjugates: their role for plant development and in the evolution of land plants. J Exp Bot 2011, 62:1757-1773.

36. Nomura K, Melotto M, He SY: Suppression of host defense in compatible plant-Pseudomonas syringae interactions. Curr Opin Plant Biol 2005, 8:361-368,

37. Svoboda J, Boland W: Plant defense elicitors: Analogues of jasmonoylisoleucine conjugate. Phytochemistry 2010, 71:1445-1449.

38. Zulak KG, Bohlmann J: Terpenoid biosynthesis and specialized vascular cells of conifer defense. J Integr Plant Biol 2010, 52:86-97.

39. Dillon VM, Overton J, Grayer RJ, Harborne JB: Differences in phytoalexin response among rice cultivars of different resistance to blast. Phytochemistry 1997, 44:599-603.

40. Grayer RJ, Kokubun T: Plant-fungal interactions: the search for phytoalexins and other antifungal compounds from higher plants. Phytochemistry 2001, 56:253-263.

41. Padmavati M, Santhivel N, Thara KV, Reddy AR: Differential sensitivity of rice pathogens to growth inhibition by flavonoids. Phytochemistry 1997 46:449-502.

42. Chen H, Wang S, Zhang Q: New gene for bacterial blight resistance in rice located on chromosome 12 identified from Minghui 63, an elite restorer line. Phytopathology 2002, 92:750-754

43. Sun X, Cao Y, Yang Z, Xu C, Li X, Wang S, Zhang Q: Xa26, a gene conferring resistance to Xanthomonas oryzae pv. oryzae in rice, encoding a LRR receptor kinase-like protein. Plant J 2004, 37:517-527.

44. Xiang Y, Cao Y, Xu C, Li X, Wang S: Xa3, conferring resistance for rice bacterial blight and encoding a receptor kinase-like protein, is the same as Xa26. Theor Appl Genet 2006, 113:1347-1355.

45. Liu Q, Yuan M, Zhou Y, Li X, Xiao J, Wang S: A paralog of the MtN3/saliva family recessively confers race-specific resistance to Xanthomonas oryzae in rice. Plant Cell Environ 2011, 34:1958-1569.

46. Agrawal GK, Yamazaki M, Kobayashi M, Hirochika R, Miyao A, Hirochika H: Screening of the rice viviparous mutants generated by endogenous retrotransposon Tos17 insertion. Tagging of a zeaxanthin epoxidase gene and a novel ostatc gene. Plant Physiol 2001, 125:1248-1257.

47. Zhu G, Ye N, Zhang J: Glucose-induced delay of seed germination in rice is mediated by the suppression of ABA catabolism rather than an enhancement of ABA biosynthesis. Plant Cell Physiol 2009, 50:644-651.

48. Koga H, Dohi K, Mori M: Abscisic acid and low temperatures suppress the whole plant-specific resistance reaction of rice plants to the infection of Magnaporthe grisea. Physiol Mol Plant Pathol 2005, 65:3-9.

49. Seo YS, Chern M, Bartley LE, Han M, Jung KH, Lee I, Walia H, Richter T, Xu X Cao P, Bai W, Ramanan R, Amonpant F, Arul L, Canlas PE, Ruan R, Park CJ, Chen X, Hwang S, Jeon JS, Ronald PC: Towards establishment of a rice stress response interactome. PLoS Genet 2011, 7:41002020.

50. Shen X, Yuan B, Liu H, Li X, Xu C, Wang S: Opposite functions of a rice mitogen-activated protein kinase during the process of resistance against Xanthomonas oryzae. Plant J 2010, 64:86-99.

51. Shen X, Liu H, Yuan B, Li X, Xu C, Wang S: OsEDR1 negatively regulates rice bacterial resistance via activation of ethylene biosynthesis. Plant Cell Environ 2011, 34:179-191.

52. Qiu D, Xiao J, Xie W, Liu H, Li X, Xiong L, Wang S: Rice gene network inferred from expression profiling of plants overexpressing OsWRKY13, a positive regulator of disease resistance. Mol Plant 2008, 1:538-551.

53. Xiao W, Liu H, Li Y, Li X, XU C, Long M, Wang S: A rice gene of de novo origin negatively regulates pathogen-induced defense response. PLOS ONE 2009, 4:e4603

54. Chen Z, Agnew JL, Cohen JD, He P, Shan L, Sheen J, Kunkel BN: Pseudomonas syringae type III effector AvrRpt2 alters Arabidopsis thaliana auxin physiology. Proc Natl Acad Sci USA 2007, 104:20131-20136.

55. Hasegawa M, Mitsuhara I, Seo S, Imai T, Koga J, Okada K, Yamane H, Ohashi Y: Phytoalexin accumulation in the interaction between rice and the blast fungus. Mol Plant Microbe Interact 2010, 23:1000-1011.

56. Wilderman PR, Xu M, Jin Y, Coates RM, Peters RJ: Identification of synpimara-7,15-diene synthase reveals functional clustering of terpene synthases involved in rice phytoalexin/allelochemical biosynthesis. Plant Physiol 2004, 135:2098-2105.

57. Shimizu T, Jikumaru Y, Okada A, Okada K, Koga J, Umemura K, Minami E, Shibuya $\mathrm{N}$, Hasegawa M, Kodama O, Nojiri H, Yamane H: Effects of a bile acid elicitor, cholic acid, on the biosynthesis of diterpenoid phytoalexins in suspension-cultured rice cells. Phytochemistry 2008, 69:973-981.

58. Scheffler BE, Reddy A, Hoffmann I, Wienand U: Chalcone synthase CDNA from rice (Oryza sativa). Plant Physiol 1995, 109:722-722.

59. Winkel-Shirley B: Biosynthesis of flavonoids and effects of stress. Curr Opin Plant Biol 2002, 5:218-223.

doi:10.1186/1746-4811-8-2

Cite this article as: Liu et al:: A convenient method for simultaneous quantification of multiple phytohormones and metabolites: application in study of rice-bacterium interaction. Plant Methods 2012 8:2.

\section{Submit your next manuscript to BioMed Central and take full advantage of:}

- Convenient online submission

- Thorough peer review

- No space constraints or color figure charges

- Immediate publication on acceptance

- Inclusion in PubMed, CAS, Scopus and Google Scholar

- Research which is freely available for redistribution

Submit your manuscript at www.biomedcentral.com/submit
C Biomed Central 